SANTOS, D. C.; EGEA, M. B.; ANDRADE, C. B. J.; SILVA, D. G. Perfil nutricional de usuários de um Spa sob os efeitos de uma dieta hipocalórica. Revista de Agricultura Neotropical, Cassilândia-MS, v. 3, n. 3, p. 52-56, jul./set. 2016.

\title{
Perfil nutricional de usuários de um Spa sob os efeitos de uma dieta hipocalórica
}

\section{Daiane Costa dos Santos ${ }^{1}$, Mariana Buranelo Egea ${ }^{1}$, Cristina Beozzo Junqueira de Andrade ${ }^{2}$, Daniella Gonçalves Silva ${ }^{2}$}

\author{
${ }^{1}$ Instituto Federal de Educação, Ciência e Tecnologia Goiano, Campus Rio Verde, Rio Verde, Goiás. E-mail: daianesantos- \\ rv@hotmail.com, mariana.egea@ifgoiano.edu.br \\ ${ }^{2}$ Universidade de Rio Verde, Rio Verde, Goiás. E-mail: silvianut@yahoo.com.br, dnutricao@yahoo.com.br
}

Recebido: 15/04/2016; Aceito: 07/07/2016.

\section{RESUMO}

Como busca de tratamentos para redução de peso existem alguns programas de SPA onde se tem um tratamento de reeducação alimentar restritiva aliada com a atividade física. O presente trabalho teve o intuito de verificar a perda de peso corporal de usuários de um SPA sob os efeitos de uma dieta hipocalórica em um período de seis dias de internação. Os dados foram coletados através de avaliação do índice de massa corporal, circunferência da cintura e percentual de gordura corporal por bioimpedância elétrica. Trata-se de estudo longitudinal descritivo onde a amostra foi composta por 30 usuários internos de um SPA, sendo $66,7 \%$ do sexo feminino e $33,3 \%$ do sexo masculino. Os dados obtidos foram analisados pelo teste $\mathrm{T}$ student, com confiabilidade de $\mathrm{p}<0,05 \%$. Os resultados mostraram que houve um efeito positivo para a perda de peso em ambos os sexos, sendo que no sexo feminino, a redução de peso, o percentual de gordura e a diminuição em relação a circunferência da cintura foi mais significativa.

Palavras-chave: obesidade, redução calórica, hábitos alimentares.

\section{Nutritional users profile of a spa under the effects of a hypocaloric diet}

\begin{abstract}
As a search of treatments for weight reduction are some SPA programs which has a treatment of restrictive eating habits combined with physical activity. The main purpose was to verify the effects of a hypocaloric diet on weight loss through evaluating the body mass index, waist circumference and body fat percentage by bioelectrical impedance analysis. The study was conducted through a descriptive study where the sample was composed of 30 internal users of a SPA $66.7 \%$ female and $33.3 \%$ male. This obtained data was analyzed through the T student test with reliability of $p<0.05 \%$. The results showed that there was a positive effect on weight loss in both sexes, but on the woman, the weight loss, the fat percentage and reduction of the waist line was more significant.
\end{abstract}

Key words: obesity, calorie reduction, eating habits 


\section{Introdução}

Mudanças no padrão alimentar brasileiro vem desencadeado alterações fisiológicas na população gerando um aumento de sobrepeso e consequentemente obesidade, contribuído com o surgimento das doenças crônicas não transmissíveis que são tratadas como problemas de saúde pública (COUTINHO et al., 2008; FERREIRA; MAGALHÃES, 2006; SOUZA, 2010). Dados recentes revelaram a prevalência mundial de obesidade mais do que duplicou entre 1980 e 2014 e, neste último ano, mais de 1,9 milhões de adultos com 18 anos ou mais apresentavam excesso de peso, dos quais 600 milhões estavam obesos (BRASIL, 2009).

De acordo com o Instituto Brasileiro de Geografia e Estatística (IBGE) cerca de 82 milhões de pessoas apresentaram o IMC igual ou superior que 25, indicando sobrepeso ou obesidade, e dentre elas o excesso de peso é maior em pessoas do sexo feminino (58,2\%), do que no sexo masculino $(55,6 \%)$ (ABESO, 2015).

A obesidade é uma doença crônica definida como um acúmulo excessivo de tecido adiposo em um nível que compromete a saúde dos indivíduos (WHO, 2000). Alguns dos fatores que levam a obesidade são destacadas: alimentação hipercalórica, depressão, ansiedade, desequilíbrio energético e o déficit de atividade física. A quantidade e tamanho das células de gordura, a distribuição regional de gordura corporal são fatores que levam a obesidade e são determinados geneticamente (LAQUATRA et al., 2005; OLIVEIRA, 2014).

A presença da obesidade é considerada fator de risco para várias dislipidemias, como hipertensão, diabetes, cardiopatias, cânceres, e outras doenças músculoesqueléticas. Existe uma exigência da sociedade quanto a estética e a aparência pessoal, e isto leva os obesos a se sentirem extremamente insatisfeitos influenciando a sua qualidade de vida, longevidade do indivíduo e com isso, muitos deles recorrem a possíveis tratamentos (CARVALHO, 2009).

Entre diversos tratamentos para redução de peso existe o SPA, que é um local destinado a tratamentos estéticos, educação alimentar e atividade física. A origem da palavra SPA veio do Latim sallus per aquam e significa cura pela água ou tratamento em água. Neste espaço, o tratamento alimentar, normalmente segue dietas hipocalóricas utilizando alimentos de baixa ação energética facilitando a perda de peso (EVANGELISTA, 2005).

Dietas hipocalóricas são estabelecidas mediante aporte calórico de aproximadamente 10 a $20 \mathrm{kcal} / \mathrm{dia}$ por $\mathrm{kg}$ de massa corporal e devem ser prescritas com a finalidade de fornecer não mais que $20 \%$ de calorias provenientes de gorduras, composta por nutrientes essenciais como carboidratos em pequenas quantidades, lipídeos em baixa percentagem e de origem vegetal e, a proteína animal deve ser derivada de carnes magras
(EVANGELISTA, 2005). Também, é característico da dieta uma maior quantidade de fibras por promover sensação de saciedade e pelo fato de possuir baixo aporte calórico e neste mesmo sentido, são utilizadas fórmulas especiais, designadas como alimentos dietéticos ou light. Quando utilizadas em maior período, podem ser prescritas suplementação de algumas vitaminas e minerais para recuperação do equilíbrio energético (GUEDES, 2002).

A perda de peso adquirida pela dieta isoladamente leva a melhorias de todo quadro patológico, mas a inclusão de um programa de exercícios físicos torna mais eficiente a ação da dieta, pois favorece o controle metabólico. Quando associados, a dieta hipocalórica e treinamento físico regular é possível atingir a redução de medidas corpóreas e da massa corporal, sendo que, apesar de uma redução em todos os depósitos de gordura, há uma prevalência na região abdominal. Estudos sugerem que isso se deve ao fato de que no tecido adiposo existem células ricas em receptores $\beta 3$ adrenérgicos, que são mais suscetíveis à lipólise (LAQUATRA, 2005; TROMBETA, 2003).

Desta forma, o objetivo deste trabalho foi avaliar o perfil nutricional dos pacientes no sistema de internato em um SPA da cidade de Rio Verde (Goiás).

\section{Material e Métodos}

Este trabalho trata se de um estudo descritivo, realizado em um SPA situado na zona rural da cidade de Rio Verde-GO. O SPA era composto por equipe multidisciplinar formada por nutricionista, educador físico, esteticista e fisioterapeuta. A amostra foi composta por 30 pacientes maiores de 18 anos que permaneceram no mínimo seis dias sob dieta hipocalórica com seis refeições diárias sendo elas: desjejum (120 kcal), colação (50 kcal), almoço (200 $\mathrm{kcal})$, lanche (80 kcal), jantar (200 kcal) e ceia (50 kcal), totalizando de $600 \mathrm{kcal}$ a $800 \mathrm{kcal} / \mathrm{dia}$. Todos frequentaram a academia realizando atividades aeróbicas. Este trabalho foi aprovado pelo Comitê de Ética em Pesquisa da Fundação de Ensino Superior de Rio Verde sob o parecer 031/2012.

Durante os seis dias de avaliação, os pacientes realizaram o desjejum às $07 \mathrm{~h} 00 \mathrm{~min}$, seguidos de caminhada por 30 minutos, alongamento e ginástica localizada. A colação foi servida às $10 \mathrm{~h} 00 \mathrm{~min}$ e após, os pacientes realizaram hidroginástica por 40 minutos. $\mathrm{O}$ almoço foi servido no período das $12 \mathrm{~h} 00 \mathrm{~min}$ e $13 \mathrm{~h} 00 \mathrm{~min}$, o lanche as $15 \mathrm{~h} 00 \mathrm{~min}$. Após o lanche era oferecido nova sessão de hidroginástica por 40 minutos. $\mathrm{O}$ jantar era servido às $18 \mathrm{~h} 00 \mathrm{~min}$ e a ceia às $21 \mathrm{~h} 00 \mathrm{~min}$. $\mathrm{O}$ tratamento estético era de escolha do paciente sendo ofertada massagem, drenagem e outros tratamentos utilizando aparelhos estéticos. 
Foi realizado no momento da internação anamnese e avaliação antropométrica, os pacientes foram identificados por números aleatórios. A anamnese consistia em informações pessoais como data de nascimento, sexo, raça, histórico de situação de saúde pessoal e de antecedentes familiares, histórico de peso e avaliação antropométrica (peso, altura circunferência de cintura, \% de tecido adiposo) e histórico alimentar onde foram coletados frequência de consumos de alimentos.

A avaliação antropométrica foi realizada no dia da internação e seis dias após a internação. Todas as medições foram realizadas no turno da manhã, em situação de conforto ambiental e em observância às recomendações prévias para a realização dos exames. Para a avaliação da massa corporal utilizou-se balança mecânica (Filizolla, Guarulhos SP, Brasil), com capacidade para $300 \mathrm{~kg}$. O paciente foi colocado ereto, braços estendidos ao longo do corpo e calcanhares juntos ao lado de uma parede sem rodapé de onde foi aferida a altura utilizando estadiômetro vertical. A altura e a massa foram utilizadas para o cálculo do Índice de Massa Corporal $($ IMC $)=$ massa $/(\text { altura })^{2}($ ANDRADE et al, 2014).

A composição corporal foi medida em aparelho Body Fat monitor (Omron BF 300, Lakeview Parkway Vernon Hills, Illinois, EUA), seguindo as recomendações do fabricante. Este dispositivo realiza a Análise de Impedância Bioelétrica (BIA) e determina a quantidade de gordura corporal e sua percentagem pela massa do corpo. Este procedimento é usado para medir a resistência elétrica dos tecidos através do envio de uma corrente elétrica baixa que não é perceptível pelo paciente avaliado. A classificação da percentagem de gordura obtida por este analisador sugerida pelo fabricante foi utilizada, sendo: baixo - homens com $10 \%$; normal - de $10 \%$ a $19 \%$; sobrepeso - de $20 \%$ a $24 \%$; e obesidade - acima de $25 \%$. Para mulheres $20 \%$ é considerado baixo; de $20 \%$ a $29 \%$ : normal; de $30 \%$ a 34\%: sobrepeso; e acima de $35 \%$ considerado como obesidade. A avaliação da circunferência da cintura (CC) foi realizada após a expiração do paciente com a blusa/camiseta levantada na sua curvatura natural, que consiste do ponto médio entre a décima costela e a crista ilíaca, ou seja, dois dedos acima do umbigo (WHO, 2000, LIMA, 2011).

Os dados obtidos foram lançados e tabulados em Excel (Microsoft, 2010), e submetidos a análise estatística por teste $\mathrm{t}$ de Student com confiabilidade de $\mathrm{p}$ $<0,05 \%$, utilizando o programa Bioestat 5.0 (ZAR, 1999). Estes valores foram comparados com os valores de referência da Organização Mundial da Saúde (WHO, 2000). Todos os participantes concordaram com a pesquisa e assinaram o TCLE.

\section{Resultados e Discussão}

Observou-se que embora a obesidade seja maior em pessoas do sexo masculino, a procura por tratamentos foi maior por pessoas do sexo feminino. Resultado semelhante foi relatado por um estudo realizado em estudo no SPA na cidade de Itapema (Santa Catarina), onde, em uma amostra de 554 pacientes, 70\% eram do sexo feminino e somente $30 \%$ do sexo masculino (SAVI et al; 2000).

Considerando a referência da Organização Mundial da Saúde (WHO, 2000), os pacientes avaliados na entrada no SPA foram classificados em sobrepeso, obesidade I, obesidade II e obesidade II conforme Figura 1. Verificou a prevalência de sobrepeso em $50 \%$ das mulheres, seguido de obesidade II (25\%), enquanto para o sexo masculino a obesidade I foi prevalente $(40 \%)$, seguido obesidade III $(30 \%)$ e não foi encontrado em ambos os sexos indivíduo eutrófico.

$\mathrm{Na}$ Tabela 1 está apresentada a relação do estado nutricional, IMC de entrada e saída e a perda de peso dos pacientes de ambos os sexos avaliados neste trabalho.

Dos 30 pacientes internos no SPA, 66,7\% eram sexo feminino e $33,3 \%$ do sexo masculino. Durante a entrevista, observou-se que 29 entrevistados (96,67\%) participaram ou fizeram algum tratamento para redução de peso em SPA. Isso corrobora com os dados de Guedes (2002) onde $70 \%$ dos indivíduos que fazem parte de programas de redução de peso, ao final de um ano recuperam o peso corporal. Essa situação evidencia que a busca pelo controle do peso corporal é complexa e não se limita à simples redução de seus valores. É necessário auxiliar os pacientes a estabelecerem modificações comportamentais, procurando interferir de maneira efetiva e permanente nos hábitos de vida. Independente do tratamento proposto, a reeducação alimentar é fundamental, uma vez que, através dela, reduz-se a ingesta calórica total e o ganho calórico decorrente (TROMBETA, 2003; SCHENEIDER, 2007).

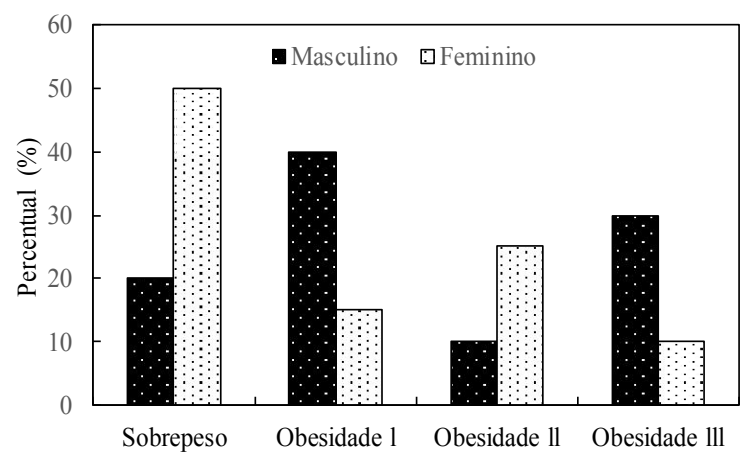

Figura 1. Prevalência de pacientes de acordo com o estado nutricional no início do internato. 
Tabela 1. Relação do estado nutricional de pacientes de um SPA, com a média de idade, IMC de entrada e saída, e perda de peso de ambos os sexos.

\begin{tabular}{|c|c|c|c|c|}
\hline \multirow{2}{*}{ Estado Nutricional } & \multirow{2}{*}{ Idade (anos) } & \multicolumn{2}{|c|}{ IMC $\left(\mathrm{kg} / \mathrm{m}^{\mathrm{a}}\right)$} & \multirow{2}{*}{ Perda de Peso (kg) } \\
\hline & & Entrada & Saída & \\
\hline & & Masculino & & \\
\hline Sobrepeso & $54 \pm 33,54$ & $26,53 \pm 0,085$ & $25,81 \pm 0,30$ & $2,25 \pm 1,34$ \\
\hline Obesidade I & $68,5 \pm 8,54$ & $33,64 \pm 1,48$ & $32,46 \pm 1,29$ & $1,55 \pm 0,13$ \\
\hline Obesidade II* & 40 & 35,71 & 34,78 & 3,80 \\
\hline \multirow[t]{2}{*}{ Obesidade III } & $24,66 \pm 9,60$ & $49,34 \pm 2,87$ & $45,44 \pm 4,24$ & $5,50 \pm 2,13$ \\
\hline & & Feminino & & \\
\hline Sobrepeso & $50,4 \pm 8,32$ & $28,63 \pm 1,11$ & $27,67 \pm 1,05$ & $2,42 \pm 0,02$ \\
\hline Obesidade I & $50,33 \pm 11,84$ & $32,90 \pm 2,00$ & $31,72 \pm 2,10$ & $3,07 \pm-0,29$ \\
\hline Obesidade II & $48,8 \pm 14,82$ & $37,59 \pm 1,77$ & $36,50 \pm 1,73$ & $2,84 \pm 0,50$ \\
\hline Obesidade III & $40,0 \pm 14,14$ & $41,05 \pm 0,17$ & $39,59 \pm 0,35$ & $3,65 \pm-1,20$ \\
\hline
\end{tabular}

*No sexo masculino foi encontrado apenas um indivíduo com obesidade grau II, não sendo possível fazer a média para avaliação.

Ao relacionar a perda de peso e os valores de IMC de entrada após o $6^{\circ}$ dia de internação de ambos os sexos, verificou que se uma perda de peso significativa $(\mathrm{p}<0,005)$, sendo mais expressiva no sexo masculino $(p=0,001)$. Houve perda de peso nos diferentes grupos, no entanto, essa resposta foi maior nos grupos com obesidade grau III. Estes dados demostraram eficácia do tratamento proposto.

A Figura 2 apresenta a relação entre a perda de peso e a faixa etária dos pacientes e em ambos os sexos ela diminui com o aumento da faixa etária. Dentre vários dos fatores que pode estar relacionado a esta condição um deles seria, devido à diminuição de $2 \%$ da taxa de metabolismo basal a cada década, tornando-se mais lento e dificultando a perda de peso (LAQUATRA, 2005).

$\mathrm{Na}$ Tabela 2 está apresentada a CC e percentual de gordura dos pacientes avaliados. Quanto a $\mathrm{CC}$ no sexo masculino, observou-se uma média de 114,90 $\pm 18,12$ $\mathrm{cm}$, e no sexo feminino $98,80 \pm 9,19 \mathrm{~cm}$, e em ambos os casos, os valores estão acima do recomendado pela WHO (2000).

A medida da $\mathrm{CC}$ é reconhecida como simples indicador da obesidade central e risco para doenças crônicas não transmissíveis, sendo por essa razão considerada no diagnóstico da síndrome metabólica (INTERNATIONAL DIABETES FEDERATION, 2006).

Quando avaliado estatisticamente pelo Test $t$, a redução da $\mathrm{CC}$ apresentou uma diferença significativa em ambos os sexos, com uma significância maior no sexo feminino $(p<0,0001)$.
Com relação ao percentual de gordura para os homens, foi observada uma média de $37,73 \pm 15,52 \mathrm{~kg}$, e um percentual de perda de 1,97 $\pm 2,67 \%$ (Figura 2), sendo maior do que o encontrado nas mulheres 35,62 \pm $9,14 \mathrm{~kg}$ e $1,44 \pm 1,16 \%$, respectivamente. A perda em relação ao percentual de gordura foi mais significativa entre as mulheres $(p=0,0001)$ do que entre os homens $(\mathrm{p}=0,0222)$. Essa heterogeneidade na distribuição da obesidade abdominal entre os sexos é consistente com os resultados descritos por alguns autores que, sugerem que maior prevalência em mulheres é atribuída a concentração de gordura corporal em virtude das gestações, diferenças hormonais e ocorrência do climatério (PINHO, 2013; VELOSO; SILVA, 2010). Assim, nestes casos onde o acúmulo de gordura foi maior, quando o paciente é submetido ao tratamento em SPA, espera-se uma maior perda de gordura.

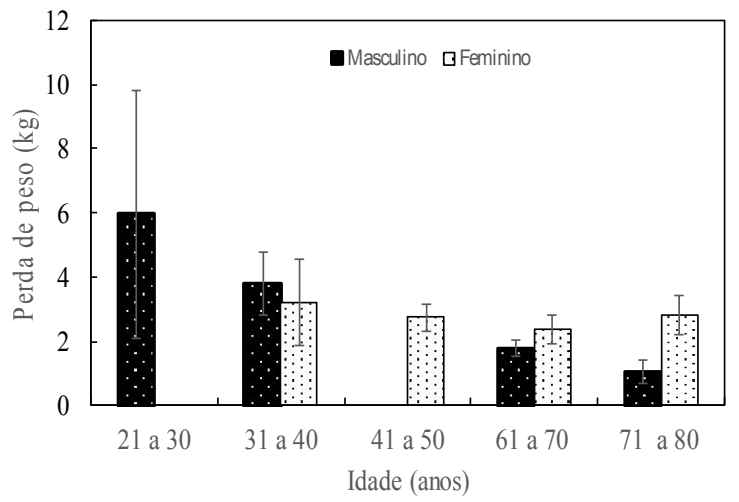

Figura 2. Demonstrativo de perda de peso em relação à média de idade dos pacientes de um SPA de Rio Verde, Goiás.

Tabela 2. Média da circunferência de cintura e percentual de gordura de pacientes de um SPA situado em Rio Verde, Goiás

\begin{tabular}{|c|c|c|c|c|}
\hline $\begin{array}{l}\text { Circunferência da cintura } \\
(\mathrm{cm})\end{array}$ & Padrão (cm) & Redução de centímetros & $\%$ de gordura em $\mathrm{kg}$ & $\begin{array}{l}\text { Redução de \% de } \\
\text { gordura }\end{array}$ \\
\hline \multicolumn{5}{|c|}{ Masculino } \\
\hline $114,90 \pm 18,13$ & $\square 102$ & $5,40 \pm 3,86$ & $37,73 \pm 15,52$ & $1,97 \pm 2,67$ \\
\hline \multicolumn{5}{|c|}{ Feminino } \\
\hline $98,80 \pm 9,19$ & $\square 88$ & $4,10 \pm 2,05$ & $35,62 \pm 9,14$ & $1,44 \pm 1,16$ \\
\hline
\end{tabular}




\section{Conclusões}

Conclui-se que a dieta hipocalórica, aliada ao exercício físico e aos tratamentos estéticos no SPA foi eficiente para a perda de peso corporal no período de 6 dias em ambos sexos, sendo que nas mulheres a perda de peso, o percentual de perda de gordura e a diminuição em relação a circunferência da cintura foi mais significativa do que nos homens. Vale lembrar que em programas de redução de peso corporal deverá ser exaltada a ética na conscientização do indivíduo, a alcançar modificações comportamentais na esperança de se estabelecerem novos hábitos alimentares e práticas de atividade física.

\section{Referências Bibliográficas}

ABESO. ASSOCIAÇÃO BRASILEIRA PARA O ESTUDO DE OBESIDADE E DA SÍNDROME METABÓLICA. Diretrizes: Federação Latino-Americana de Endocrinologia e Federação Latino-Americana de Obesidade. 2014 - 2015. São Paulo-SP: 2015. (Série científico) Disponível em: $<$ http://www.abeso.org.br/uploads/downloads/91/572a58480c bd8.pdf.> Acesso em: 19 de maio. 2016.

ANDRADE, F.T.; MARTINS, M.C.C.; SANTOS, M.A.P.; TORRES-LEAL, F.L.; FERREIRA, A.H.C. Estimativa do percentual de gordura utilizando o IMC. Revista Brasileira de Obesidade, Nutrição e Emagrecimento, São Paulo-SP, v. 8., n. 47., p. 142-147, 2014.

BRASIL/IBGE. INSTITUTO BRASILEIRO DE GEOGRAFIA E ESTATÍSTICA. POF 2008-2009: Desnutrição cai e peso das crianças brasileiras ultrapassa padrão internacional. Brasília-DF: 2009. (Série Legislação Brasileira) Disponível em: $<$ http://www.ibge.gov.br/home/presidência/noticias/noticia_vi sualiza.php?id_noticia $=1699 \&$ id_pagina=1> Acesso em: 19 de maio. 2016.

CARVALHO K.M.B. Obesidade e síndrome metabólica. In CUPPARI, L: Nutrição nas doenças crônicas não transmissíveis. $1^{\text {a }}$ ed. Barueri-SP: Manole, cap. 3., 2009. p. 23-119.

COUTINHO, J.G.; GENTIL, P.C.; TORAL, N. A desnutrição e obesidade no Brasil: o enfrentamento com base na agenda única da nutrição. Caderno Saúde Pública, Rio de JaneiroRJ, v. 24, n. 2, p. 332-340, 2008.

EVANGELISTA, J. Alimentos especiais. In: Evangelista, J. Alimentos: Um estudo abrangente. $3^{\mathrm{a}}$ ed. São Paulo-SP: Atheneu, 2005, p.74.

FERREIRA, V.A.; MAGALHÃES, R. Obesidade no Brasil: tendências atuais. Revista Portuguesa de Saúde Pública, Lisboa, v. 24, n. 2, p.71-81, 2006.

GUEDES, D.C. Programas de controle do peso corporal: Atividade física e nutrição. Revista Mineira de Educação Física. Viçosa-MG, v. 10, n.1, p. 64-90, 2002.
IDF. INTERNATIONAL DIABETES FEDERATION. The IDF consensus worldwide definition of the metabolic syndrome. Brussels-Belgium: IDF, 2006, p.16.

LAQUATRA, I. Nutrição para o controle de peso. In: MAHAN, L. K. S. Alimentos, nutrição \& dietoterapia. São Paulo-SP: Roca, 2005. cap. 24. p. 534-565.

LIMA, C.G; BASILE, L.G; SILVEIRA, J.Q.; VIEIRA, P.M; OLIVEIRA, M.R.M. Circunferência da cintura ou abdominal? Uma revisão crítica dos referenciais metodológicos. Revista Simbio-Logias, São Paulo-SP, v.4, n.6, p.108-131, 2011.

OLIVEIRA, A. P. S. V; SILVA, M. M. Fatores que dificultam a perda de peso em mulheres obesas de graus I e II. Revista Psicologia e Saúde, Rio de Janeiro-RJ, v. 6, n. 1, p. 74-82, 2014.

PINHO, C. P. S; DINIZ, A. S; ARRUDA, I. K. G; FILHO M. B; COELHO, P. C; SEQUEIRA, L. A. S; LIRA, P. I. C.. Prevalência e fatores associados à obesidade abdominal em indivíduos na faixa etária de 25 a 59 anos do Estado de Pernambuco, Brasil. Caderno Saúde Pública, Rio de JaneiroRJ, v. 29, n.2, p. 313-324, 2013.

SAVI, C. B; SALLES, R. K; ZENI, L. A. Z. R; FIATES, G. M. R. Dietas Hipocalóricas em Internação: Perda de Peso em Seis Dias. Arquivo Brasileiro de endocrinologia e metabolismo, São Paulo-SP, v. 44, n. 6, p. 497-501, 2000.

SCHENEIDER, C; VERAS, A; NASCIMENTO, M.B; LIBERALI, R. Efeitos de um programa de intervenção nutricional sobre a composição corporal de e os hábitos alimentares de obesos em spa/ SC. Revista Brasileira de Obesidade, Nutrição e Emagrecimento. São Paulo-SP, v.1, n.1, p. 90-101, 2007.

SOUZA, E.B. Transição nutricional no Brasil: análise dos principais fatores. Cadernos UniFOA, Volta Redonda-RJ, v. 1, n. 13, p. 49-53, 2010.

TROMBETTA, I.C. Exercício físico e dieta hipocalórica para o paciente obeso: vantagens e desvantagens. Revista Brasileira Hipertensão, São Paulo-SP, v. 10, n.2, p. 130-133, 2003.

VELOSO, H. J. F; SILVA, A. U. M. Prevalência e fatores associados à obesidade abdominal e ao excesso de peso em adultos maranhenses. Revista Brasileira Epidemiologia, Rio de Janeiro-RJ, v. 13, n. 3, p. 400-412, 2010.

ZAR, J. H. Bioestatistical analysis. 4 ed. New Jersey: Prentice Hall, 1999. 663p.

WHO. WORLD HEALTH ORGANIZATION. Obesity: preventing and managing the global epidemic. Report of a World Health Organization Consultation. Geneva: World Health Organization, 2000. p. 256. 